\title{
Three fields used in lubricating oil for demulsification
}

\author{
Ye Peng ${ }^{a}$, Tao Liu ${ }^{b}$ \\ School of Mechanical and Electronical Engineering, Lanzhou University of Technology, Lanzhou \\ 730050, China \\ apyaigb@163.com, ${ }^{\mathrm{b}}$ liutao1971@lut.cn
}

Keywords: demulsification; three-field coupling; emulsion oil; lubricating oil

Abstract. Lubricating oil can be easily polluted by water, thereby negatively affecting the lubricating property. A single technological method is difficult to develop for efficient demulsification and dehydration of emulsified lubricating oil. In this paper, we propose the combination of swirl centrifugal field made by hydrocyclone, pulse electric field, and vacuum temperature field to achieve our research goals. The three-field coupling demulsification-dehydration device was tested in two modes. Results indicate that the use of this device is more energy efficient and faster compared with the traditional vacuum heating method.

\section{Introduction}

Lubricating oil is prone to pollution during storage, transportation, filling, and use and is then converted into emulsified oil [1]. The emulsified oil affects the physical and chemical properties of the lubricating oil, thereby leading to a decreased level of oiliness and lubrication failure [2]. Common dehydration purification methods of waste lubricating oil include sedimentation and centrifugation etc., but all have shortages [3]. For emulsified lubricating oils polluted by water, the use of a single physical technique, such as vacuum heating, for rapid and efficient demulsification is difficult. Only the optimal combination of two or more unit operations can ensure successful separation, but this strategy is difficult to employ for conventional technologies [3]. However, this coupling or integrated technology is the main technique used in the development of demulsification technologies for emulsified oil.

This article mainly discusses the integration or coupling of three common physical fields, namely, hydrocyclone, pulse electric, and vacuum temperature fields, to achieve fast and efficient demulsification-dehydration of emulsified lubricating oil.

\section{Three-field Coupling Demulsification and Dehydration}

The three-field coupling demulsification device of the emulsified lubricating oil was designed, and the working principle diagram is shown in Fig.1. The demulsification device mainly consists of two parts, namely, the hydrocyclone (structure parameters need to be optimized) with a set of copper electrodes fixed on the parallel part of the entrance and the back-end vacuum heating demulsification portion. Under the effect of screw pump, solid impurities (such as metal particles and sand) in oil can be filtered through the strainer. The emulsion droplets from the strainer coalesce and increase in size because the pulse electric field is fused in the hydrocyclone, and the oil-water separation is rapidly accomplished under the effect of the hydrocyclone field. The oil from the overflow port of hydrocyclone contains a small amount of water, which is difficult to be removed by using the pulse electric and hydrocyclone fields only. After being heated, the oil flows into the vacuum tank. Under the action of nitrogen blowing, the water vapor in the oil is then fully released and the oil is protected from oxidation. After entering the condenser, water vapor condenses into water. In addition, the system sets the inner circulation function to increase the time of the oil to flow through the vacuum tank and to deeply purify the oil. 


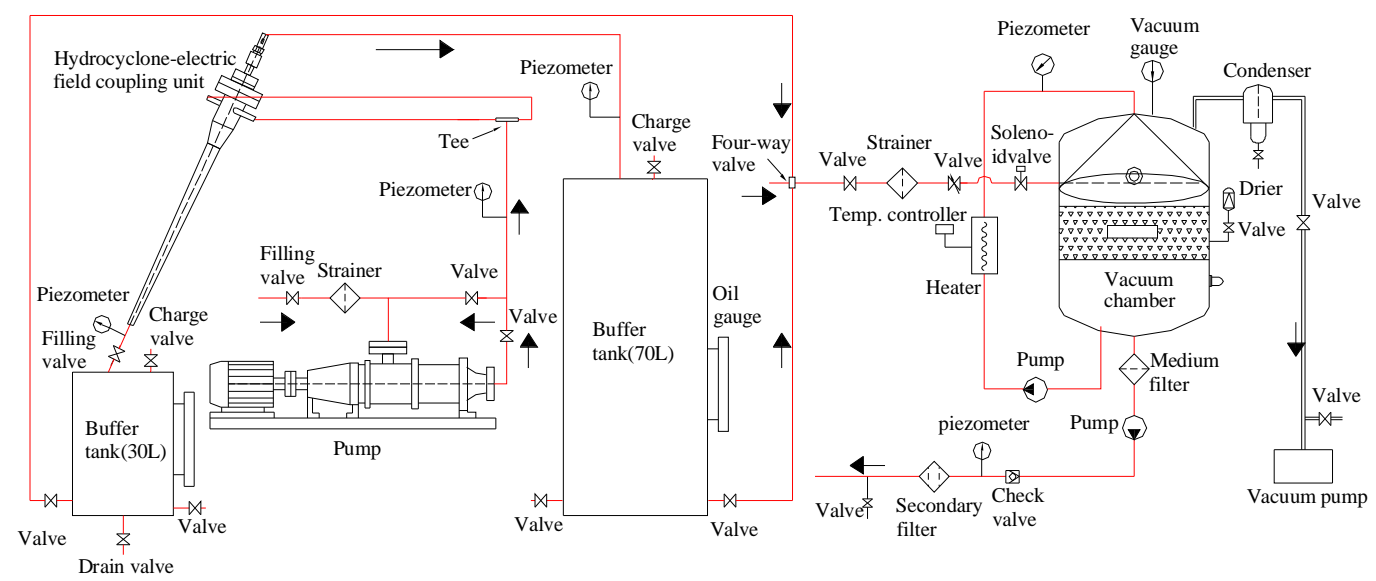

Fig.1 Diagram of the working principle of three-field coupling demulsification-dehydration device

Electric Field-Hydrocyclone Field Coupling Unit. When the gravity field is replaced with the hydrocyclone field, the unit couples with the hydrocyclone and high-voltage pulse electric fields. As such, the high-voltage pulse electric field is used to coalescence small emulsion droplets within a short time, and the hydrocyclone field is used to separate the settled and coalesced droplets within a short time. Through this process, the efficiency of demulsification-dehydration of emulsified oil can be improved and waste oil can be efficiently and rapidly dehydrated [4].

The main part of the unit is the hydrocyclone with a double-cone section, and the tangential double-inlet is designed to stabilize the flow field (shown as in Fig.1) [5]. The overflow port is designed to be bigger than that of the deoiling hydrocyclone because the latter is classified as a dehydration type. Thus, the oil phase can rapidly flow out. At the same time, the cone vertex angle of hydrocyclone is increased to accelerate oil discharge. The overflow pipe has an elongation quantity in the straight pipe of the hydrocyclone and is made up of copper. The overflow pipe, which is insulated with the tube body through the insulating material, is used as the positive pole of the high-voltage electric field, whereas the straight pipe of hydrocyclone is used as the negative pole. Thus, a high-voltage electric field is formed in the straight pipe of hydrocyclone.

Vacuum Demulsification Dehydration Unit. As shown in Fig.1, after the emulsified oil flows through the electric field-hydrocyclone field coupling unit, most water flows out from the underflow pipe, whereas the oil with a small amount of water flows from the overflow pipe and then into the vacuum heating unit under negative pressure. The unit adopts the efficient vacuum separation tower and the appropriative oil atomizer. Compared with traditional models, the vacuum heating unit presents higher separation efficiency and separation degree, thereby considerably improving the vacuum separation effect.

In Fig.1, the emulsified oil, which flows from the overflow pipe and then into the strainer through the filling valve, thereby filtering bulky mechanical impurities. The emulsified oil then flows into the vacuum separation chamber through the oil-taking control valve and the oil-taking solenoid valve. After the pressure is increased by the oil circulating pump, the emulsified oil is heated by the heater and the temperature gradually increases. After stabilizing temperature by using the temperature controller, an oil mist cover is formed because of the positive pressure at the top of the vacuum chamber by the atomizer. This mist can prevent the rise of oil bubbles in the vacuum separation chamber and can avoid the injection of the vacuum pump of oil during the operation. Thus, antifoaming is automatically prevented. After atomization, the oil reenters the efficient separation tower, in which an oil film is again formed. The developed area of the oil film significantly increases. The water in the oil rapidly transforms from liquid into vapor. When nitrogen is at the bottom of the vacuum separation chamber, the water vapor in oil is removed and expelled from the top. Under the oil drain pump, the pressure of oil after dehydration changes from negative to positive, and the oil flows from the oil discharge outlet through the check valve and the fine filter. After one or more circulations, clean oil is generated. 


\section{Experiment}

Device Parameters and Experiment. The relevant operation performance parameters of the device are shown in Table 1.

Table 1 Relevant operation performance parameters of the device

\begin{tabular}{ccccc}
\hline Nominal flow rate & $\begin{array}{c}\text { Inlet pressure of } \\
\text { hydrocyclone }\end{array}$ & Total power of pump & Heating power & Pulse voltage/frequency \\
\hline $20 \mathrm{~L} / \mathrm{min}$ & $0.6 \mathrm{MPa}$ & $4.5 \mathrm{~kW}$ & $20 \mathrm{~kW}$ & $9 \mathrm{kV} / 60 \mathrm{HZ}$ \\
\hline
\end{tabular}

In the device performance test, the emulsified 46\# steam turbine oil with a water content of $15 \%$ was selected as the experimental oil sample. In the experiment, the device was used to conduct the demulsification-dehydration treatment of the emulsified oil in two modes. The first mode involves pretreatment by applying the pulse electric field-hydrocyclone field coupling unit. Subsequently, circulatory deep demulsification dehydration was performed through the vacuum heating unit. The second mode involves no pretreatment, where the emulsified oil directly flows into the vacuum heating unit to conduct demulsification dehydration. The oil moisture contents of the overflow port of hydrocyclone and the oil drainage port of vacuum heating unit were determined in the first test mode to investigate the processing capacity and effectiveness of the pulse electric field-hydrocyclone field coupling unit and the device. Moreover, the unit energy consumption and the unit time consumption of the device in the two test modes were then compared.

Results and Discussion. In the two test modes, the experiment was conducted three times and the unit energy and time consumption were compared, as shown in Table 2 . $W_{v}$ is the unit energy consumption of the vacuum treatment, $W_{m}$ is the unit energy consumption of the multi-field coupling treatment, $t_{v}$ is the unit time consumption of the vacuum treatment, and $t_{m}$ is the unit time consumption of the multi-field coupling treatment.

Table 2 Results of device test

\begin{tabular}{ccccc}
\hline Terms & $\begin{array}{c}\text { Rate of water in the } \\
\text { overflow of } \\
\text { cyclone(\%) }\end{array}$ & $\begin{array}{c}\text { Rate of water in the } \\
\text { outlet of device } \\
(\mathrm{ppm})\end{array}$ & $\frac{\mathrm{W}_{v}-\mathrm{W}_{m}}{\mathrm{~W}_{v}} \times 100 \%$ & $\frac{\mathrm{t}_{v}-\mathrm{t}_{m}}{\mathrm{t}_{v}} \times 100 \%$ \\
\hline Firstly & 5.5 & 120 & 80 & 80.4 \\
Secondly & 6.3 & 145 & 78 & 78.5 \\
Thirdly & 5.8 & 135 & 83 & 83.4 \\
Mean values & 5.9 & 133 & 80 & 80.7 \\
\hline
\end{tabular}

In Table 2, the initial moisture content of the experimental emulsified oil sample is $15 \%$. After the treatment of the pulse electric field-hydrocyclone field coupling unit, the moisture content ranges from $5 \%$ to $6 \%$. This finding indicates that the unit exhibits a good demulsification dehydration effect, and the pretreatment for the emulsified oil with high water content is active and effective. The oil moisture content of the oil drainage port of the vacuum device is lower than $150 \mathrm{ppm}$. Regardless if the three-field coupling demulsification-dehydration or the traditional vacuum heating dehydration was used, the oil moisture content is maintained at low levels after the treatment with the vacuum heating unit. However, in the two test modes, when the oil moisture contents are equal after the treatment, both unit energy and unit time consumption significantly differ from each other. The unit energy and unit time consumption of the three-field coupling dehydration mode are reduced by $80 \%$ and $80.7 \%$, respectively, compared with those of the single vacuum heating dehydration mode. Table 2 shows that the three-field coupling demulsification and dehydration device exhibit the same effect as the single vacuum heating dehydration unit in the treatment effect of the emulsified oil. Nevertheless, the former is superior in terms of unit energy and unit time consumption. The pulse electric field-hydrocyclone field coupling unit presents an evident effect in the treatment of emulsified lubricating oil and has a large contribution in reducing the subsequent processing time of the vacuum heating unit. 


\section{Conclusions}

(1) When the high-voltage pulse electric field, hydrocyclone field, and vacuum heating field are integrated, the original single treatment process can be modified and a new demulsification and dehydration technology of waste oil is achieved.

(2) The electric field-hydrocyclone field coupling unit is important in improving the treatment efficiency of the whole device.

(3) Compared with single vacuum heating dehydration, the use of the three-field coupling demulsification device is superior in terms of energy consumption and processing time.

\section{Acknowledgements}

This work was partially supported by grants from the Chinese National Natural Science Foundation (Grant No. 21206204), projects of CQ CSTC (Grant No. cstc2015jcyjA90018 and cstc2014yykfB90002) and the Chinese Postdoctoral Science Foundation (Grant No. 2015M572640XB).

\section{References}

[1] G. Y. Xu, M. Lu. China Resources Comprehensive Utilization, 12(2003) 14-16. In Chinese

[2] H. W. Yang, Y.W. Fei, J.G. Hu. Lubricant Oil, 21(2006) 9-11. In Chinese

[3] T. C. Li, S.P. Cheng. Chinese Hydraulics \& Pneumatics, 1(2003)41-42. In Chinese

[4] X.L. Yang. Drop dynamics of w/o emulsion under the combination of centrifugal field and pulsed electric field [D], China University of Petroleum, China, 2009. In Chinese

[5] H.Y. Du. CFD simulation of double cone type oil-water separator[D], Southwest Petroleum Institute, China, 2005. In Chinese 